\title{
EFFECT OF DIFFERENT ANTICOAGULANTS IN DETERMINING METFORMIN HYDROCHLORIDE LEVELS IN HUMAN PLASMA USING HIGH-PERFORMANCE LIQUID CHROMATOGRAPHY
}

\author{
YAHDIANA HARAHAP*, LISTA RORO MARSUDI, SUNARSIH \\ Faculty of Pharmacy, Universitas Indonesia, Depok 16424, Indonesia. Email: yahdiana03@yahoo.com
}

Received: 08 June 2018, Revised and Accepted: 09 August 2018

ABSTRACT

Objective: This study aimed to develop and validate an analytical method and to determine the effect of different anticoagulants for analyzing metformin $\mathrm{HCl}$ level in human plasma.

\begin{abstract}
Methods: Analysis was performed with high-performance liquid chromatography method using a photodiode array detector set at 234 nm, a $\mathrm{C}_{18}$ SunFire ${ }^{\mathrm{m}}$ column $(5 \mu \mathrm{m}, 250 \mathrm{~mm} \times 4.6 \mathrm{~mm})$, a temperature of $40^{\circ} \mathrm{C}$, a mobile phase comprising $10 \mathrm{mM}$ sodium dodecyl sulfate and $10 \mathrm{mM}$ phosphate buffer in water-acetonitrile $(60: 40 \mathrm{v} / \mathrm{v})$ at $\mathrm{pH} 7.0$, with a flow rate of $1.0 \mathrm{~mL} / \mathrm{min}$, and atorvastatin calcium as the internal standard. Plasma extraction was performed through protein precipitation using human plasma (300 $\mu \mathrm{L})$ and acetonitrile $(600 \mu \mathrm{L} ; 1: 2 \mathrm{v} / \mathrm{v})$. The method was linear at a concentration range of $20.0-5000.0 \mathrm{ng} / \mathrm{mL}$, with $\mathrm{r}>0.9998$.
\end{abstract}

Results: The stability and recovery of metformin $\mathrm{HCl}$ in human plasma were not different between citrate with ethylenediaminetetraacetic acid (EDTA) and heparin with EDTA as anticoagulants. However, a significant difference in the peak area response ratio (p<0.05) was observed between the three anticoagulants.

Conclusion: The validation results for the three types of plasma anticoagulants met validation requirements based on the European Medicines Agency bioanalytical guidelines of 2011 for accuracy and precision, selectivity, calibration curve linearity, dilution integrity, carry-over, and stability.

Keywords: Anticoagulant, Citrate, Ethylenediaminetetraacetic acid, Heparin, Human plasma, Validation.

(C) 2018 The Authors. Published by Innovare Academic Sciences Pvt Ltd. This is an open access article under the CC BY license (http://creativecommons. org/licenses/by/4. 0/) DOI: http://dx.doi.org/10.22159/ijap.2018.v10s1.31

\section{INTRODUCTION}

Metformin $\mathrm{HCl}$ is a biguanide drug that lowers blood sugar levels by decreasing the production of hepatic glucose (gluconeogenesis) and increasing the action of insulin (stimulating glycogenesis). Metformin is generally used for patients with Type 2 diabetes and has been proven as an effective antihyperglycemic drug, thus reducing the risk of death [1]. Furthermore, metformin may lower plasma glucose levels by reducing glucose absorption from the intestine [2]. The maximum metformin levels in plasma $\left(\mathrm{C}_{\max }\right)$ have been reported to be $1600 \pm 380 \mathrm{ng} / \mathrm{mL}$. Metformin is also used for various critical conditions; thus, it requires a definite response and bioequivalence (BE) tests for a copy or generic drugs $[3,4]$. BE testing is conducted using pharmacokinetic studies involving the determination of plasma drug levels. Therefore, determining metformin concentrations in human plasma are necessary for pharmacokinetic studies [5].

The measurement of drug concentrations in the blood, serum, or plasma is considered the best approach to obtain a pharmacokinetic profile of a given drug [6]. In pharmacokinetic studies, plasma as biological matrix is usually used for analysing analyte and metabolite in plasma. Plasma drug levels are so low that sensitive and selective measurement techniques are required [7]. Plasma is an often-used biological matrix and is obtained through blood sampling using anticoagulants. Thus, one of the most important factors in obtaining plasma is the type of anticoagulant used [8]. Anticoagulants that are frequently used in blood drug analysis include ethylenediaminetetraacetic acid (EDTA), heparin, and citrate $[8,9]$. The type of anticoagulant commonly used in Indonesia for obtaining plasma is the citrate form as citric phosphate dextrose adenine because it has the ability to extend the plasma storage time [10]. Both citrate and EDTA work by binding calcium ions for the prevention of protein coagulation. In contrast, heparin has mechanisms as an antithrombin activator [8]. These differences between the type of anticoagulant used during drug analysis may affect small molecules, metabolic profiles, and clinical parameters of the drug to be analyzed [9]. Indeed, the choice of anticoagulant is an important factor because some ions $\left(\mathrm{Na}^{+}\right.$or $\left.\mathrm{K}^{+}\right)$in the plasma have anticoagulant properties (e.g., Na-citrate) and others such as K-EDTA can cause either the ion suppression or ion enhancement in analyzing analyte or metabolite, which may affect the matrix during analysis [11].

A bioanalysis method should be validated before analysis for the method to be accurate and reliable [7]. Based on the European Medicines Agency (EMEA) 2011, full validation is performed for each $1^{\text {st }}$ time analysis method or new compound drugs [12]. If an anticoagulant is used for analysis, then full validation should be performed using the same anticoagulant. For minor change conditions such as anticoagulant changes in an analysis method that was once validated, a partial validation is acceptable

Several methods are available for analyzing metformin $\mathrm{HCl}$ level in the plasma, including high-performance liquid chromatography (HPLC), which is the most widely used technique for the analysis of drugs in biological matrices $[13,14]$. In general, the use of a photodiode array (PDA) detector provides advantages for sensitive and selective HPLC systems during the analysis of drugs and their metabolites in biological matrices $[15,16]$. Using these principles, various analytical methods for determining plasma metformin levels have been widely developed and accepted [5,17-20].

Research into the effects of different anticoagulants on the determination of metformin $\mathrm{HCl}$ levels in vitro using HPLC has been limited; however, this information is important to better understand the pharmacokinetics of metformin in human plasma and its analysis. Therefore, in this study, we developed an in vitro metformin $\mathrm{HCl}$ analytical method for human plasma, with a focus on evaluating the 
influence of commonly used anticoagulants, such as EDTA, citrate, and heparin, on specific analysis parameters, such as stability, recovery, chromatogram interference, and peak analytical response.

\section{METHODS}

\section{Equipment}

A set of HPLC devices (Waters 2965; Separation Module) consisting of pumps, an autosampler, C18 columns (Waters, SunFire ${ }^{\mathrm{TM}} 5 \mu \mathrm{m}$; $250 \mathrm{~mm} \times 4.6 \mathrm{~mm}$ ), PDA detectors (Waters 2996) set at $234 \mathrm{~nm}$ wavelength, with data processing on a computer were used. In addition, other equipment used in this study included a $\mathrm{pH}$ meter (Eutech $\mathrm{pH}$ 510), vacuum tubes and Vacutainers with K3EDTA anticoagulant (Vacuette ${ }^{\circledR}$ ), vacuum tubes with Li-Heparin anticoagulant (Vacuette ${ }^{\circledR}$ ), blood collection tubes (BD Vacutainer ${ }^{\circledR}$ Beckton, Dickinson and Company), analytical scales (Acculab), eluent filter (Whatman), busting/ degasser (Elmasonic $\mathrm{S} 60 \mathrm{H}$ ), microcentrifuge (Spectrafuge $16 \mathrm{M}$ ), a $-80^{\circ} \mathrm{C}$ freezer (Biomedical Labtech Deep Freezer), a $-20^{\circ} \mathrm{C}$ freezer (Biomedical Labtech Deep Freezer), refrigerator set to $4^{\circ} \mathrm{C}-8^{\circ} \mathrm{C}$, vortex (Maxi Mix II), Eppendorf micropipettes (Socorex), centrifuge tubes, sample cups, blue tips, yellow tips, filter paper (Perkin Elmer), and various glassware.

\section{Materials}

The reagents used in this study were of analytical grade and included metformin $\mathrm{HCl}$ (United States Pharmacopeia [USP]), calcium atorvastatin (USP), methanol pro HPLC (Merck), orthophosphoric acid (Merck), acetonitrile pro HPLC (Merck), sodium dodecyl sulfate (SDS) (Merck), sodium dihydrogen phosphate (Merck), sodium hydroxide (Merck), aquabidestilata, and human plasma (Indonesian Red Cross). Blood samples were obtained directly from healthy subjects.

\section{Stock solution}

Standards of metformin $\mathrm{HCl}$ and atorvastatin calcium were each weighed to obtain $10 \mathrm{mg}$. The compounds were then placed into a $10-\mathrm{mL}$ measuring flask. From this solution, $1 \mathrm{~mL}$ was then further diluted into $10 \mathrm{~mL}$. Water was then added to the metformin $\mathrm{HCl}$ solution, while methanol was added to the calcium atorvastatin solution to prepare test concentrations of the stock solution.

\section{Optimization of chromatographic conditions}

The analysis was conducted using HPLC equipped with detector PDA at wavelength $(\lambda) 234 \mathrm{~nm}, \quad$ C18 column (Waters, SunFire ${ }^{\mathrm{TM}} 5 \mu \mathrm{m} ; 250 \mathrm{~mm} \times 4.6 \mathrm{~mm}$ ). $10 \mathrm{mM}$ SDS and $10 \mathrm{mM}$ phosphate buffer in water-acetonitrile $(60: 40)$ were used as the mobile phase, and was adjusted to $\mathrm{pH} 7.0$ with $98 \%$ orthophosphoric acid. The column temperature was set to $40^{\circ} \mathrm{C}$. The flow rate was adjusted from $0.8 \mathrm{~mL} / \mathrm{min}$ to $1.0 \mathrm{~mL} / \mathrm{min}$ over a 10 -min analysis time.

\section{System suitability test}

A mixture of $10 \mathrm{mg} \mu \mathrm{g} / \mathrm{mL}$ of metformin $\mathrm{HCl}$ and $10 \mu \mathrm{g} / \mathrm{mL}$ of calcium atorvastatin was injected as $50 \mu \mathrm{L}$ into a HPLC equipped with PDA detector instrument at a wavelength of $234 \mathrm{~nm}$. The injection results were recorded and counted for the coefficient of variation $(\% \mathrm{CV})$ of the 5 times injection. The recorded parameters included the area and peak retention time.

\section{Sample preparation}

Sample preparation of metformin $\mathrm{HCl}$ in plasma was performed by protein precipitation. A $300-\mu \mathrm{L}$ aliquot of plasma containing a concentration of metformin $\mathrm{HCl}$ was added to the sample cup, and $50.0 \mu \mathrm{L}$ of standard calcium atorvastatin $10 \mu \mathrm{g} / \mathrm{mL}$ was added. $600 \mu \mathrm{L}$ acetonitrile was then added as a protein precipitator. The mixture was vortexed for $3 \mathrm{~min}$ to extract the metformin $\mathrm{HCl}$ from the plasma. The mixture was then centrifuged to precipitate proteins at 13,000 rpm for $10 \mathrm{~min}$. Aliquots of $1000 \mu \mathrm{L}$ were centrifuged and transferred into vials. Aliquot $(50.0 \mu \mathrm{L})$ was then injected into the HPLC system.

\section{Validation of bioanalysis methods}

In this study, the validation of the metformin $\mathrm{HCl}$ method in plasma was performed with the use of different types of anticoagulants:
Citrate, EDTA, and heparin. Full validation was performed using plasma with the citrate anticoagulant and monitored for selectivity, carryover parameters, lowest quantity limit measurement (lower limit of quantification [LLOQ]), linearity of the calibration curve, accuracy, precision and recovery, dilution integrity, and stability. Partial validation was performed using EDTA and heparin for the plasma, with minimum parameters of accuracy and precision. However, in this study, we also performed recovery and stability tests on plasma EDTA and heparin to determine the influence of the type of anticoagulant used. Validation of the analytical methods was evaluated according to the EMEA guidelines 2011 for the validation of bioanalysis methods.

\section{RESULTS AND DISCUSSION}

\section{Optimization of conditions for the analysis method}

The analysis method we used in this study was based on an HPLC method. The analysis conditions used C18 column (Waters, SunFire ${ }^{\mathrm{TM}} 5 \mu \mathrm{m}$; $250 \mathrm{~mm} \times 4.6 \mathrm{~mm}$ ). In addition, $10 \mathrm{mM}$ SDS and $10 \mathrm{mM}$ phosphate buffer in water-acetonitrile $(60: 40)$. The mobile phase was adjusted to pH 7.0 with $98 \%$ orthophosphoric acid, the column temperature was set to $40^{\circ} \mathrm{C}$, with a flow rate of $1.0 \mathrm{~mL} / \mathrm{min}$ over a 10 -min analysis time. Calcium atorvastatin was selected as an internal standard due to its similar physicochemical properties, including $\mathrm{pKa}$ value. The analytical conditions resulted in the good separation of the targeted compound. The retention time of metformin hydrochloride was $5.4081 \mathrm{~min}$ and that of calcium atorvastatin was $7.021 \mathrm{~min}$ (Fig. 1). The runtime was quite short at $10 \mathrm{~min}$, which was considered as an optimum condition.

\section{System suitability test}

$\mathrm{CV}$ requirements should be $<2.0 \%$. The results obtained from each system suitability test consistently yielded $\mathrm{CV}$ values $<2.0 \%$. In addition to $\mathrm{CV}$, tailing factor was close to 1 (1.698), $\mathrm{N}$ (theoretical plates) was high and height equivalent to the theoretical plate (HETP) values were close to zero. Our results indicated that the $N$ (theoretical plate) was 2698.88 and the HETP value was 0.0092 . The analysis time for each injection was $10 \mathrm{~min}$. The system suitability test was performed every time to perform the analysis on a daily basis and to confirm the parameters met the requirements of the validation process.

Based on the results of the optimization of the centrifugation time, we obtained the highest area under curve of metformin $\mathrm{HCl}$ with a centrifugation time of $10 \mathrm{~min}$ at a speed of 13,000 rpm with an area of 115,432 . Therefore, the extraction conditions were considered optimal for extracting the analytes from plasma for the types of anticoagulants used (plasma citrate, plasma heparin, and EDTA plasma).

\section{Validation of bioanalysis methods}

\section{LLOQ (citrate plasma)}

In this study, the measurement of the LLOQ value was performed by creating an analytical calibration curve with range concentration of $20.0-5000 \mathrm{ng} / \mathrm{mL}$. In this process, the concentration of $20.0 \mathrm{ng} / \mathrm{mL}$ becomes the temporary limit of LLOQ and the replication is carried out for 5 times. The values of percentage difference and $\mathrm{CV}$ at a

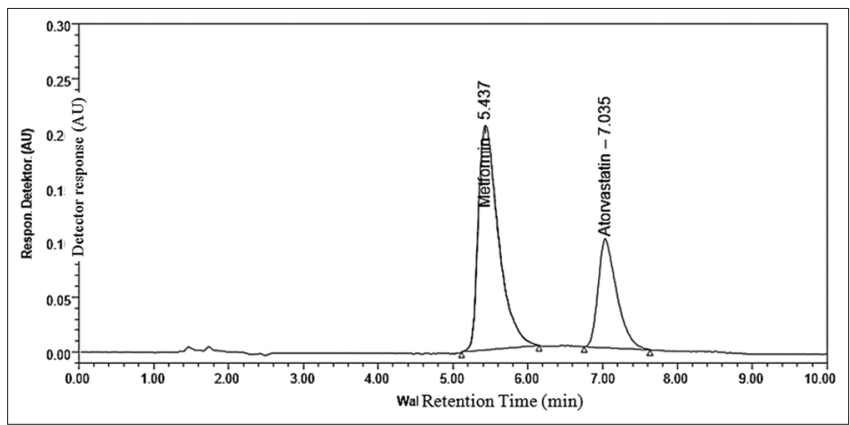

Fig. 1: Chromatogram of the system suitability test. Optimization of sample preparation 
concentration of $20.0 \mathrm{ng} / \mathrm{mL}$ still met the requirement of not more than $20 \%$; thus, the concentration met the criteria of accuracy and precision.

\section{Selectivity test (citrate plasma)}

In this study, we get the percentage difference values of LLOQ is $3.11-19.77 \%$, which still fulfilled the requirement $(< \pm 20 \%)$ and yielded a coefficient of $4.05 \%$. In addition, at the time of analysis, the retention and standard plasma chromatogram for the blank showed some interference, but this is not significant. Interference for metformin $\mathrm{HCl}$ was $2.36-2.61 \%$ and for calcium atorvastatin it was $0.02-0.04 \%$, indicating the method used in this study met the selective criteria.

\section{Calibration curve (citrate plasma, heparin, and EDTA)}

Calibration curve analysis was performed regarding the linearity parameter $(r>0.9990)$, accuracy (\% difference) $> \pm 15 \%$, with the exception of that the LLOQ concentration should $< \pm 20 \%$. The calibration curve for plasma citrate yielded a linear regression equation of $y=0.0016+0.0002 x$, with $r=0.9999$. The heparin plasma calibration curve yielded a linear regression equation of $y=0.0041+0.0002 x$, with $r=0.9996$. The EDTA calibration curve yielded a linear regression equation of $y=0.0022+0.0002 x$, with $r=0.9995$, where $x$ is the concentration of metformin $\mathrm{HCl}(\mu \mathrm{g} / \mathrm{mL})$ and y is peak area ratio (PAR) between the response of the analyte area and the inner standard.

\section{Test accuracy, precision (citrate plasma, heparin, and EDTA)}

For the measurement of accuracy using plasma citrate, we obtained a percentage difference value with the range of $+5.85-+18.93 \%$ for the LLOQ concentration and a range of $-14.67-14.31 \%$ for the QC concentration. For the measurement of intraday precision using plasma citrate, we obtained a CV value $<4.65 \%$ for the LLOQ concentration and $<8.56 \%$ for QC samples. For the precision interday values, we obtained a CV value of $<7.97 \%$ for both the LLOQ and QC. Both intraday and interday accuracy and precision values still met the requirements for the LLOQ $< \pm 20 \%$ and QC samples $< \pm 15 \%$. In measuring the accuracy of heparin plasma, the values of the percentage difference ranged from $-15.26 \%$ to $+10.50 \%$ for the LLOQ concentration, and ranged from $-14.64 \%$ to $+13.88 \%$ for plasma QC. The measurement of the intraday precision for heparin plasma obtained a CV value $<9.50 \%$ for the LLOQ and QC concentrations. For the precision interday evaluation in plasma heparin, we obtained a CV value $<8.46 \%$ for both LLOQ and QC concentrations. For the measurement of accuracy in plasma EDTA, we obtained a value of percentage difference of $-15.59-+14.21 \%$ for the LLOQ concentration, with a range of $-10.30-+14.61 \%$ for QC samples. For the intraday precision measurement in plasma EDTA, we obtained CV values $<11.24 \%$ for LLOQ concentrations and QC samples. For the precision interday values, we obtained CV values $<11.37 \%$ for both LLOQ concentrations and QC samples.

\section{Dilution integrity test (plasma citrate)}

To perform the dilution integrity test, we made an analyte solution in plasma with the concentration exceeding the LLOQ, which was equal to $7500.0 \mathrm{ng} / \mathrm{L}$. We then diluted this solution by a of $1 / 2$ and $1 / 4$. The results of the dilution were tested for accuracy and precision, both for intraday and interday parameters. The values of the $\%$ diff were $-4.34-7.63 \%$ for dilution $1 / 2$ times and percentage difference of $-5.86-11.55 \%$ for dilution $1 / 4$ times. As for the value of the intraday variation coefficient $(\mathrm{KV})$, we obtained a value of $<7.53 \%$ with the interday $\mathrm{KV}$ value $<3.08 \%$ for both the dilution $1 / 2$ and $1 / 4$ times. The accuracy and precision values of the dilution still fulfilled the precise criteria; thus, the dilution described in this study was considered validated.

\section{Carry-over (plasma sitrat)}

In this study, the percentage of followings for the analyte was $8.10 \%$, while for the standard it was $0.03 \%$. The percentage of the follow-up still met the requirements for analytes at $<20 \%$, while for standards it is $<5 \%$. This indicates that, during the analysis process, there was no meaningful carry-over between injections.
Stability test of metformin $\mathrm{HCl}$ and calcium atorvastatin stock solution

The results of the stability test in this study indicated a value of percentage difference for metformin stock solution $\mathrm{HCl}$ and calcium atorvastatin for $24 \mathrm{~h}$ at room temperature of $-0.75--0.16 \%$, with a value of percentage difference of $-1.78-0.20 \%$ after 3 days storage. Therefore, the standard analytical and raw stock solutions used for $24 \mathrm{~h}$ and stored at room temperature were stable for at least 31 days in storage at $4^{\circ} \mathrm{C}$.

\section{Comparison of chromatograms of plasma citrate, heparin, and EDTA}

There were no significant differences between the three chromatogram forms. We did observe some interference with plasma citrate at the 4-min retention time, but this did not interfere with the analytical process (Fig. 2).

\section{Comparison of metformin HCl stability in plasma citrate, heparin, and EDTA}

To compare the stability of metformin $\mathrm{HCl}$ using different anticoagulants, including citrate, heparin, and EDTA, we determined if each stability test fulfilled the requirement that at both low and high concentration, quality control dictates percentage difference values of $\pm 15 \%$. Therefore, during the bioanalysis process, the storage of metformin $\mathrm{HCl}$ in plasma citrate, heparin, and EDTA in the analysis room was still stable for 31 days and can be used for the analysis process at $-20^{\circ} \mathrm{C}--80^{\circ} \mathrm{C}$.

Comparison of the peak analytical response of plasma citrate, heparin, and EDTA

Given the different characteristics of anticoagulants, including differing physicochemical properties, plasma $\mathrm{pH}$, and ionic strength, it is necessary to observe the chromatogram form and peak area response generated by the analyte and the standard with each anticoagulant type. In this study, we compared the PAR at the concentrations of Quality Control Low (QCL), Quality Control Medium (QCM) and Quality Control High $(\mathrm{QCH})$ in each plasma sample. The analysis was done statistically to observe any differences. Based on the statistical analysis of PAR for each plasma sample, we obtained a $\mathrm{p}<0.05$ (Kruskal-Wallis), which was good for the concentrations of QCL, QCM, and QCH. Therefore, the hypothesis that there is no significant difference was rejected because the $p$ value we obtained was $<0.05$, indicating a significant difference between the PAR values generated from plasma citrate, heparin, and EDTA.

Comparison of the absolute recovery value and the modified recovery with plasma citrate, heparin, and EDTA

In the comparative analysis of modified recovery values using the plasma matrix as a comparison (not the standard solution, which is used for the absolute recovery), we obtained statistical results for QCL

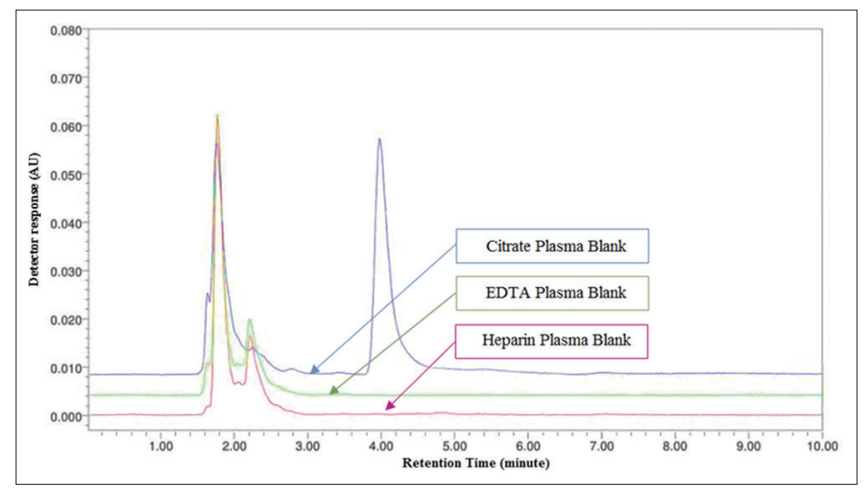

Fig. 2: Overlay chromatogram of plasma citrate blanks, heparin, and ethylenediaminetetraacetic acid 
and $\mathrm{QCH}$ concentrations at $\mathrm{p}=0.602$ and $\mathrm{p}=0.129$, respectively. Since $\mathrm{p}>0.05$ (ANOVA, least significant difference post hoc), the hypothesis was accepted, indicating no significant difference in recovery between plasma citrate, heparin, and EDTA.

\section{CONCLUSION}

The method developed in this study to analyze plasma concentrations of metformin $\mathrm{HCl}$ using HPLC obtained an LLOQ of $20 \mathrm{ng} / \mathrm{mL}$. The validation results for the three types of plasma anticoagulants met validation requirements based on the EMEA bioanalytical guidelines of 2011 for accuracy and precision, selectivity, calibration curve linearity, dilution integrity, carry-over, and stability.

Based on the comparison of several analytical parameters, there were no significant differences for plasma with citrate, heparin, or EDTA anticoagulants regarding stability and recovery of metformin $\mathrm{HCl}$ in plasma ( $p>0.05$, ANOVA). However, for the peak plasma response ratio of metformin $\mathrm{HCl}$ with the plasma anticoagulants citrate, heparin, and EDTA, a significant difference $(\mathrm{p}<0.05)$ was observed between plasma with the anticoagulant citrate-EDTA and plasma with the heparin-EDTA anticoagulant for low, moderate, and high concentrations. In the plasma blast chromatogram with citrate anticoagulant, there was considerable interference of plasma at a retention time $<5 \mathrm{~min}$, whereas in plasma with heparin and EDTA anticoagulants, no interference was observed.

\section{CONFLICTS OF INTEREST}

None declared.

\section{REFERENCES}

1. Dipiro J, Cecily V, Barbara GW, Terry LS. Pharmacotherapy: A Pathophysiologic Approach. $9^{\text {th }}$ ed. United States: The McGraw-Hill Companies, Inc; 2015.

2. Goodman L, Gilman A, Brunton L, Lazo J, Parker K. Goodman and Gilman's the Pharmacological Basis of Therapeutics. $1^{\text {st }}$ ed. New York: McGraw-Hill; 2006.

3. Food and Drug Administration. Metformin Hydrochloride Tablets. United States of America: Department of Health and Human Services; 2002.

4. Heinig K, Bucheli F. Fast liquid chromatographic-tandem mass spectrometric (LC-MS-MS) determination of metformin in plasma samples. J Pharm Biomed Anal 2004;34:1005-11.

5. Zarghi A, Foroutan S, Shafaati A, Khoddam A. Rapid determination of metformin in human plasma using ion-pair HPLC. J Pharm Biomed Anal 2003;31:197-200

6. Shargel L, Wu-Pong S, Yu ABC. Applied Biopharmaceutics and
Pharmacokinetics. $5^{\text {th }}$ ed. United States of America: Appeton and Lange; 2004.

7. Harahap Y. The Role of Bioanalysis in Drug Quality Assurance and Improving Quality of Patient Life. Jakarta: UI-Press; 2010.

8. Bowen RA, Hortin GL, Csako G, Otañez OH, Remaley AT. Impact of blood collection devices on clinical chemistry assays. Clin Biochem 2010;43:4-25.

9. Gonzalez-Covarrubias V, Dane A, Hankemeier T, Vreeken RJ. The influence of citrate, EDTA, and heparin anticoagulants to human plasma LC-MS lipidomic profiling. Metabolomics 2012;9:337-48

10. Ludong MM. Blood Transfusion. Jakarta: Faculty of Medicine, Universitas Tarumanegara; 2012. p. 1-11.

11. Barri T, Dragsted LO. UPLC-ESI-QTOF/MS and multivariate data analysis for blood plasma and serum metabolomics: Effect of experimental artefacts and anticoagulant. Anal Chim Acta 2013;768:118-28.

12. European Medicines Agency. Guideline on Bioanalytical Method Validation. Vol. 44. London: An Agency of the European Union; 2011.

13. AbuRuz S, Millership J, McElnay J. Determination of metformin in plasma using a new ion pair solid phase extraction technique and ion pair liquid chromatography. J Chromatogr B Analyt Technol Biomed Life Sci 2003;798:203-9.

14. Haque A, Priya S, Mohanty D, Bakshi V, Boggula N. Analytical method development and validation of amlodipine in human plasma using liquid chromatography-mass spectrometry/mass spectrometry. Asian J Pharm Clin Res 2017;11:393-7.

15. Evans G. A Handbook of Bioanalysis and Drug Metabolism. United States of America: CRC Press; 2004.

16. Pawar AK. Development and validation of UV spectrophotometric and reversed phase-high performance liquid chromatography-PDA methods for the estimation of alogliptin benzoate. Asian J Pharm Clin Res 2017;9:282-7.

17. Porwal P, Talele G. Development of validated HPLC-UV method for simultaneous determination of metformin, amlodipine, glibenclamide and atorvastatin in human plasma and application to protein binding studies. Bull Fac Pharm 2016;55:129-139.

18. Porta V, Schramm S, Kano E, Koono E, Armando Y, Fukuda K, et al. HPLC-UV determination of metformin in human plasma for application in pharmacokinetics and bioequivalence studies. J Pharm Biomed Anal 2008:46:143-7.

19. Chhetri H, Thapa P, Van SA. Simple HPLC-UV method for the quantification of metformin in human plasma with one step protein precipitation. Saudi Pharm J 2013;22:483-7.

20. Alakhali KM. Validation method for measuring simvastatin in human plasma by HPLC-UV and its application ii study simvastatin stability in plasma and working solution. Asian J Pharm Clin Res 2017;7:131-3. 